\title{
PERCEPCIÓN MUSEOGRÁFICA DE LA BIBLIOTECA HISTÓRICA O PATRIMONIAL: PERSPECTIVAS Y REFLEXIONES EN TORNO A LOS FONDOS Y LIBROS ANTIGUOS
}

\author{
Manuel-José Pedraza-Gracia
}

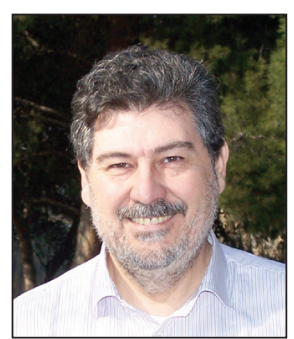

Manuel-José Pedraza-Gracia, doctor en historia por la Universidad de Zaragoza (UZ), fue bibliotecario de dicha universidad entre 1979 y 1989. Es profesor titular del Área de Ciencias de la Documentación (UZ). Ha publicado diversos trabajos científicos en materias relacionadas con la historia del libro y de las instituciones documentales, libro antiguo y tasación y valoración del libro antiguo. Ha dirigido los cursos de verano de la Universidad de Zaragoza sobre tasación, valoración y comercio del libro antiguo.

http://orcid.org/0000-0001-9686-9031

Facultad de Filosofía y Letras, Universidad de Zaragoza Depto. de Ciencias de la Documentación e Historia de la Ciencia Pedro Cerbuna, 12. 50009 Zaragoza, España pedraza@unizar.es

\section{Resumen}

Se analizan las relaciones existentes entre bibliotecas con fondos patrimoniales y museos. Se estudian especialmente las características similares del patrimonio bibliográfico y los objetos patrimoniales conservados en los museos, de las condiciones de uso y de las características de los usuarios, de la gestión de estos fondos por parte de los profesionales. Finalmente se analizan las bibliotecas que únicamente poseen este tipo de fondos.

\section{Palabras clave}

Biblioteca patrimonial, Biblioteca histórica, Museos, Patrimonio bibliográfico, Libro antiguo.

Title: Museographic view of history or cultural heritage libraries: perspectives and reflections about collections and old books

\begin{abstract}
The paper analyses the similarities between cultural heritage libraries and museums. First, books are analyzed as heritage objects and museum artifacts. Secondly, researcher's needs and interests concerning rare books are studied, as well as the knowledge and skills a librarian must possess. Finally, main functions and special features of heritage libraries are outlined.

\section{Keywords}

Heritage libraries, Museums, Bibliographical heritage, Rare books, Old books.

Pedraza-Gracia, Manuel-José (2013). “Percepción museográfica de la biblioteca histórica o patrimonial: perspectivas y reflexiones en torno a los fondos y libros antiguos". El profesional de la información, septiembre-octubre, v. 22, n. 5, pp. 440-447.
\end{abstract}

http://dx.doi.org/10.3145/epi.2013.sep.09

\section{Introducción}

Los objetos y bienes que constituyen los patrimonios de los pueblos se caracterizan por poseer un conjunto de valores de tan singular significación que llegan a conformar la identidad de esos pueblos (Arjona, 2003; Abranches, 1988; Pedraza-Gracia, 2008; Racedo et al., 2004; Unesco, 2000; 2009). Este principio -que se ha fijado claramente en la legislación del Estado Español, en alguna de las normativas de la Comunidades Autónomas como Navarra, La Rioja, País Vasco o Cataluña y en las de Iberoamérica como Perú o Venezuela, entre otras- afecta a objetos y bienes de muy diverso tipo, algunos de los cuales se custodian en los diferentes centros de información: museos, archivos y bibliotecas, e incide de diversas maneras en ellos. Las bibliotecas como custodios y como constituyentes de ese conjunto de objetos y bienes también se ven afectadas por este principio: desde el objeto -el libro o la colección-, desde el usuario -el investigador o el ciudadano interesado-, desde el bibliotecario y sus formas de actuación y gestión, y desde la propia biblioteca. Si se analizan las soluciones que se adoptan en las bibliotecas con fondos patrimoniales se aprecia que muchas de ellas son muy próximas, si no son coincidentes, con las que afec- 
tan a la pieza museística, al usuario, al conservador y al museo.

Sin embargo, esta proximidad no suele ser advertida y no existen trabajos científicos desde la biblioteconomía y la documentación que pongan en relación las bibliotecas que poseen fondos patrimoniales con los centros museísticos, lo que no quiere decir que no se hayan estudiado desde otras perspectivas.

El interés de este trabajo se encuentra en el análisis, desde una posición exclusivamente teórica, de esta posible relación entre ambos tipos centros de información: biblioteca patrimonial, histórica o de reserva, con fondos históricos, conventuales, de reserva, preciosos curiosos o raros... y museo; especialmente por lo que hace referencia a su promoción y difusión.

\section{El libro antiguo}

Cuando la biblioteca conserva entre sus colecciones y fondos conjuntos o unidades documentales de cierta antigüedad -lo que puede derivar en la posesión de valores patrimoniales contrastables-, se suele producir una disfunción entre este conjunto bibliográfico y el resto de los materiales conservados en la misma. Estas disfunciones tienen como fundamento las diferencias existentes entre el valor y uso del documento bibliográfico contemporáneo y el valor y uso de los documentos bibliográficos antiguos.

El documento editado fabricado manualmente puede ofrecer diferencias entre los distintos ejemplares de la edición. La existencia de conjuntos de ejemplares de la misma edición denominados, de forma específica, emisiones y estados tiene su origen en una casuística compleja. Si estas diferencias han sido planificadas intencionadamente, la variante recibe el nombre de emisión y si no ha sido planificada intencionalmente, el de estado (Moll, 1979; Clemente, 2003, pp. 251-253; Martín-Abad, 2004a, pp. 53-59, pp. 81-85). La identificación de la edición es aún más compleja si a la existencia de variantes se añade que la diferenciación entre las sucesivas ediciones -reediciones- no es sencilla debido a que su mención no suele ser relevante por lo que respecta a los contemporáneos del documento y no suele figurar de forma evidente en él. Todavía hay que añadir la existencia de ediciones ilegales, contrahechas, piratas, falsificadas y subrepticias (Clemente, 2003, p. 254). En consecuencia, en el proceso de producción del libro en el período de la imprenta manual se crean diferentes ediciones, no siempre sencillas de identificar, que poseen variantes, en ocasiones, complejas en su apreciación. Pero no por ello se debe obviar el trance imprescindible de la identificación (Martín-Abad, 2004b, pp. 6-12).

Independientemente de las vicisitudes de su fabricación, con el transcurso del tiempo, el libro puede sufrir las acciones del medio ambiente y las intervenciones del ser humano que introducen nuevos elementos diferenciadores: ataques biológicos, una encuadernación especialmente cuidada o rica, una indicación de posesión, la pérdida de integridad (motivada o no), la inclusión en un facticio, una anotación aclaratoria, una marca de fuego, el uso descuidado..., entre una casuística variadísima de actuaciones (superada la más drástica: la propia desaparición del documento). Así se obtienen unidades bibliográficas perfectamente diferenciables que pueden acabar denominándose por el lugar en el que son conservados o por el nombre del poseedor. La procedencia del ejemplar resulta uno de los elementos diferenciadores más esenciales y a la par uno de los principios de estudio de los fondos y bibliotecas (Pearson, 1998; MartínAbad, 2004a; Santos-Aramburo; Torres-Santo-Domingo, 2004). Algunos de estos factores inciden también y de la misma manera en los bienes patrimoniales que se custodian en museos.

\section{Existen diferencias entre el valor y uso del documento bibliográfico contempo- ráneo y el valor y uso de los documentos bibliográficos antiguos}

Por todo lo mencionado, de aquí se puede extraer una nueva consecuencia: el libro antiguo no puede tener el mismo interés de búsqueda y uso que el libro moderno, aunque se encuentren en el mismo centro. El documento moderno como documento de investigación o consulta se busca y se usa por su contenido (científico, literario, artístico, de ocio...). El documento antiguo como documento de investigación o consulta presenta características específicas:

a) En muy escasas ocasiones el libro antiguo es buscado para su lectura, para el ocio o para el estudio del contenido como ocurre con los documentos modernos. Se busca en este caso, por tanto, la obra. Este tipo de actuación responde a obras poco conocidas, aún no editadas por autores modernos o incluidas entre los millones de ejemplares digitalizados disponibles en la Red. Quizás esto es lo único que puede justificar esta actuación, ya que en la mayoría de las ocasiones no resulta pertinente-ni recomendable- usar con 
el único fin de su lectura una edición antigua de una obra cuyas ediciones modernas presentan complementos informativos que ayudan y mejoran su comprensión, aunque es cierto que en ese proceder existe un componente fetichista muy propio de la bibliofilia o, mejor, de la bibliomanía. No es comprensible usar una primera edición de las Comedias de Lope para su mera lectura o intentar estudiar en determinados niveles de aproximación a las ideas de Newton sus primeras ediciones. Sólo los expertos en determinadas materias se aproximan a estas ediciones príncipe con objeto de realizar análisis de los textos minuciosos tendentes a discernir mediante el estudio del medio los mensajes en toda su intención.

Más que una herencia de nuestros antepasados, los objetos patrimoniales son un préstamo de nuestros sucesores (Tugores; Planas, 2006)

b) En otras ocasiones el ejemplar se busca como unidad que pertenece o perteneció a un conjunto (colección, edición, variante, obra de un encuadernador concreto, producto bibliográfico de una imprenta, libros censurados...) persiguiendo, por ejemplo: la identificación de una edición, el análisis minucioso del texto de una variante, la autentificación de una anotación, una procedencia específica, la anotación de un censor, la reconstrucción de una colección disgregada, la lectura de la parte tachada por el censor, la identificación de un ex libris... (todo ello mediante la comparación con otros ejemplares del mismo u otro conjunto, de manera principal). Una de estas actuaciones es la de adscribir un ejemplar a una edición específica, muy importante cuando se trata de libro antiguo, lo que se ha denominado la identificación del ejemplar ideal, definido perfectamente por Moll (1979) como "el que ofrece el estado más perfecto de la obra, según la intención del autor, editor e impresor", que ha de permitir, por ejemplo, la correcta catalogación del documento. La identificación resulta aún más complicada en los impresos sine notis. De nuevo aquí se cuenta con la ayuda de la Red donde ya existe un número significativo de ejemplares digitalizados.

c) En otras se quiere estudiar un ejemplar determinado de una colección dotado de valores y de información estructural concreta que lo independiza y diferencia de todos los demás (sin que, como en el caso anterior, sea imprescindible que lo que busca el investigador sea el propio producto editorial). El ejemplar es estudiado por aquello que solamente él posee: una anotación marginal, una dedicatoria, un dibujo, un grabado adherido a las guardas, un cuaderno de anotaciones cosido, una miniatura... En este caso, aunque estuviese digitalizado hay aspectos que únicamente se pueden percibir desde el original. De la misma manera que hay aspectos de una obra pictórica, por ejemplo, que sólo se pueden apreciar ante ella y jamás ante una reproducción porque la materialidad de la obra trasmite también información y sentimiento; lo que no impide que se hayan creado herramientas gráficas e informáticas que facilitan el análisis pormenorizado de la obra pictórica, aunque en su mayoría son herramientas de investigación presencial y no de uso. Jan Bos (2012, p. 18) ha puesto de manifiesto la existencia de una ausencia efectiva de identidad ante la investigación entre el documento original y la copia digital, lo que se percibe de manera iconográfica en “Ceci n'est pas une pipe" de René Magritt ${ }^{1}$. Por esta causa, aquí no se pretende tratar las posibilidades que ofrecen los documentos digitalizados ni las bibliotecas digitales, que quedan para análisis posteriores.

\section{Uso, conocimiento y transmisión}

El patrimonio tiene como imperativo legal la transmisión. La identidad de un pueblo se sostiene en la existencia de un aglutinante, la cultura, que a su vez se sustenta en la existencia de bienes culturales, no siempre tangibles. Si desaparecen estos bienes desaparece ese aglutinante y con ello el pueblo como tal, como ya se argumentó en la introducción. Es ésta la raíz del genocidio cultural, ese crimen contra toda la humanidad que se produce cuando los pueblos que se apoderan de otro destruyen su patrimonio (arrasando las bibliotecas y archivos, penalizando, despreciando o prohibiendo el uso de una lengua o privándolo de sus obras artísticas, por ejemplo). Por esta misma causa, la legislación antepone la preservación al uso. Los investigadores en patrimonio aducen siempre una premisa: más que pensar que los objetos patrimoniales son una herencia de nuestros antepasados se debería tener presente que son un préstamo de nuestros sucesores (Tugores; Planas, 2006). El bibliotecario que trabaja con fondos antiguos no debería olvidarlo. La biblioteca con respecto al patrimonio bibliográfico tiene la obligación de activar ese patrimonio para su conocimiento y uso por parte del ciudadano. Se trata de dotar de su función social al patrimonio o, si se quiere, su uso social (Prats, 1997, p. 27).

Debido a las vicisitudes de su elaboración y en el transcurso del tiempo, el libro antiguo deviene en ejemplar único

La actividad de la biblioteca varía con respecto a los fondos patrimoniales. Por lo que respecta al libro moderno la biblioteca debe servir para cubrir las necesidades informativas, de formación, de ocio... de los usuarios; resulta evidente que la utilización del documento prevalece sobre la conservación, la rentabilidad de la inversión en la colección se mide por el grado de uso y satisfacción del usuario. En lo referente a los fondos y unidades documentales antiguos, la responsabilidad y el punto de mira varían de forma notable, la biblioteca es responsable del mantenimiento y conservación del bien patrimonial, de tal suerte que el uso está supeditado a la preservación. El foco cambia de manera radical.

La racionalización del uso de estos materiales en las bibliotecas se convierte entonces en un problema que se ha de resolver casi de forma cotidiana y que se presenta con dos vertientes: qué se usa y quién lo usa.

Es evidente que cuando un documento pueda resultar dañado por el uso deberá preservarse su utilización hasta ha- 
ber encontrado la manera de superar los problemas que ocasionan los desperfectos y éstos mismos. También resulta evidente que, aunque sea de forma imperceptible, cualquier grado de uso supone una degradación del documento, por lo que se puede reservar la utilización del mismo a aquellos usuarios que puedan aportar o mejorar los valores del documento o de la colección (Serrai, 1992). El problema radica, por lo tanto, en resolver la paradoja presentada anteriormente: ¿cómo accede un ciudadano a un bien que por ser patrimonial le pertenece?

Los responsables deben potenciar el acceso al conocimiento $y$ al uso de los bienes patrimoniales mediante exposiciones permanentes y temporales, préstamo para exposiciones, charlas y conferencias, jornadas de puertas abiertas, publicaciones, ediciones facsimilares y otros recursos similares. $Y$ es aquí donde se encuentra otra similitud entre el museo y la biblioteca con fondo patrimonial. Existe un uso reservado a la investigación de los documentos, ya que la investigación genera nuevo conocimiento sobre el documento estudiado (en lo que respecta a sus contenidos o a sus aspectos formales o materiales) que se supedita a unas reglas de uso estrictas encaminadas a la preservación del documento y que el investigador conoce. Pero se debe potenciar de forma decidida el conocimiento del patrimonio propio de los legítimos propietarios, que sepan de los valores patrimoniales del mismo, independientemente de su nivel de preparación y conocimiento del documento, facilitando su interpretación, lo que también es un mandato legislativo. De aquí se debe deducir que no solamente se atenta contra el patrimonio impidiendo su investigación, sino que también es un atentado el impedir su conocimiento y disfrute mediante el acceso a las actividades de difusión que informen sobre el mismo, que pongan de manifiesto su valor cultural.

En este sentido las exposiciones se muestran como uno de los mejores métodos: acercan el documento al ciudadano, le proporcionan información sobre sus valores, su trascendencia, su autor, sus características..., en suma, proporcionan un conocimiento que sólo puede redundar en aumento de interés por el documento y por su investigación.

El investigador es quien se aproxima al libro o a la biblioteca con un conocimiento del mismo de alto nivel (de la misma manera que el investigador se aproxima a una pieza en el museo), conoce su procedencia y su génesis, sabe cómo se trata el objeto y reconoce su importancia. Es un usuario profesionalizado, cuya actividad posee trascendencia para el centro, ya que sus aportaciones sobre las piezas generan nuevo conocimiento. Su producción (científica) aporta siempre nuevo(s) valor(es) añadido(s) a los documentos o piezas que posee el centro, lo que conduce a que esa producción científica sea imprescindible para el centro de investigación. De esta manera se produce un efecto dinamizador del centro y de la investigación, ya que estos nuevos conocimientos y valores añadidos producen un efecto llamada sobre otros investigadores que, a su vez, aportarán nuevo conocimiento y valores al documento y al centro, y así sucesivamente.

\section{El libro antiguo requiere un proceso de identificación imprescindible para poder llevar a cabo un tratamiento correcto}

Junto a este usuario investigador hay también un ciudadano interesado, un usuario que ansía conocer, no profesionalizado, que no suele tomar un contacto directo (físico) con el documento bibliográfico o la pieza pero que es el objeto del centro, es el destinatario de la función social del mismo, asiste a conferencias y a las exposiciones, a las jornadas de puertas abiertas, se interesa por los catálogos de exposiciones... y, con ello, se forma e informa. Es este usuario el que proporciona sentido al centro.

Especial mención merecen los jóvenes y los niños que han de ser especialmente cuidados, deben organizarse actividades propias para esas edades y visitas especialmente preparadas, con zonas lúdicas relacionadas con el centro, el libro antiguo y con recursos específicos; también en las bibliotecas históricas o patrimoniales. No obstante, se debe diferenciar estructuralmente entre los espacios y funciones relacionados con la investigación, con los dedicados a la difusión y el conocimiento, y con los dedicados a los procesos.

No puede existir un tipo de usuario sin el otro. La ley determina como una de las finalidades principales del centro que custodia bienes patrimoniales que el ciudadano los (re) conozca y disfrute como propios. Pero también es esencial que un centro de información propicie la investigación sobre sus fondos patrimoniales como método de valoración de los mismos y de difusión de los nuevos conocimientos que se obtienen como fruto de esas investigaciones.

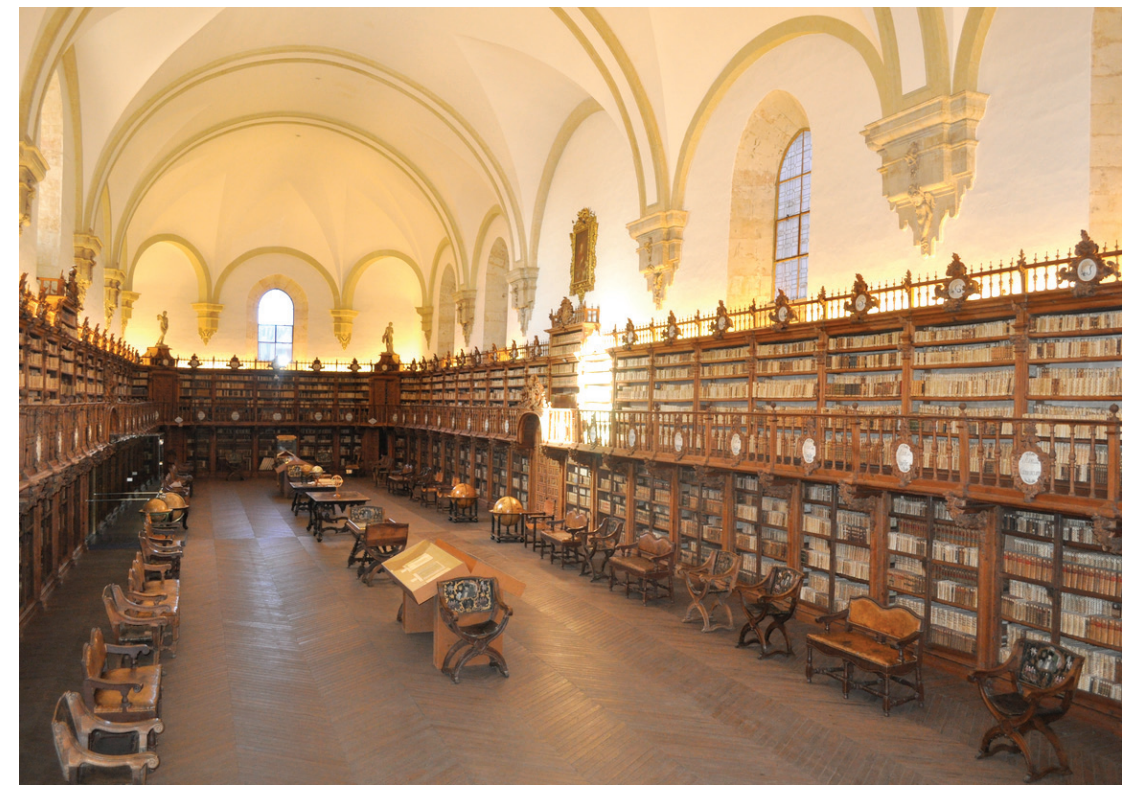

Biblioteca General Histórica, Universidad de Salamanca 


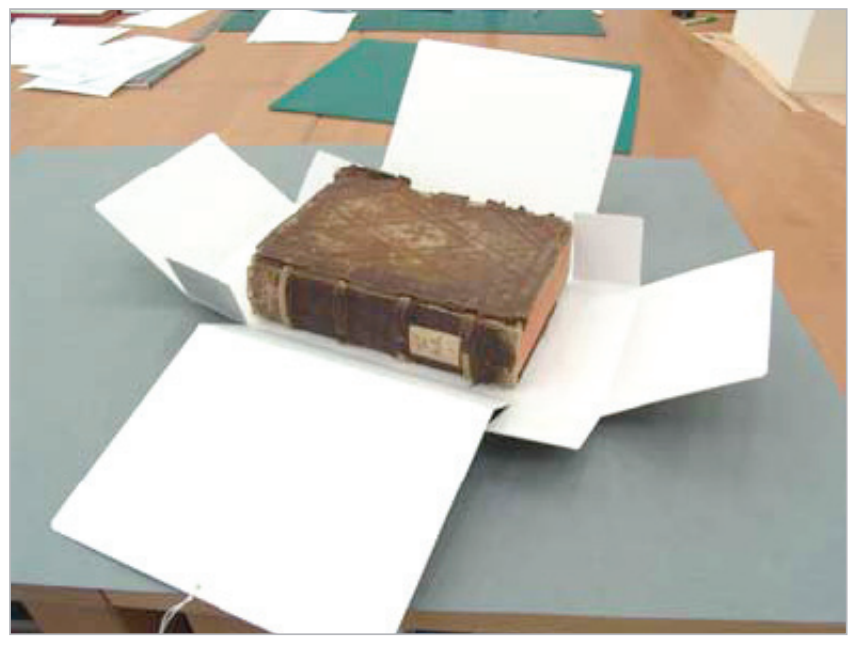

Caja de conservación (Tacón-Clavaín, 2010a)

\section{El bibliotecario}

La actividad del profesional ante los libros antiguos también posee características específicas. Son tantas y tan trascendentes las diferencias que existen entre los documentos impresos antiguos y los contemporáneos que forzosamente requieren que el profesional que se encarga de ellos haya de poseer una especialización (Richard, 2010). Por esta causa, sería necesaria una reflexión desde las instituciones que se dedican a la formación en información y documentación sobre si ofrecen las competencias y aptitudes que se precisan en estos centros. La vertiente patrimonial es demandada y tiene mucha aceptación entre los estudiantes de los centros en los que se ha cuidado y ofertado.

Las labores especializadas de selección y adquisición no son iguales a las de los libros contemporáneos, puesto que requieren el conocimiento de un mercado complejo en el que se adquieren ejemplares específicos de una edición, que presentan particularidades que hacen preferible a uno sobre los otros.

Aspectos elementales como el sellado, colocación de tejuelos o ubicación del documento en la estantería, en aras de la preservación, ofrecen algunas dificultades que no presentan los libros modernos, hasta incluso la incorporación del libro en cajas especialmente preparadas para su protección. Por todo ello son precisos conocimientos sobre preservación.

Tampoco el tratamiento es el mismo, el libro antiguo requiere una identificación previa e imprescindible: es preciso conocer qué es lo que se tiene delante para poder efectuar un tratamiento correcto. Los elementos que ofrecen ese conocimiento en los documentos antiguos pueden estar muy escondidos en el propio documento o requerir herramientas bibliográficas específicas. Sin esta actividad, que apenas afecta al libro contemporáneo, resulta imposible llevar a efecto cualquier actuación sobre un libro antiguo (restaurar, catalogar, tasar...). Se trata de una actividad múltiple y con diversas facetas que afecta a la obra, a la edición, a la variante y a aspectos formales como la encuadernación o las marcas de propiedad, entre otros. La catalogación exige, además de las normas específicas, un conocimiento profundo del producto bibliográfico antiguo y de los múltiples aspectos formales ya mencionados.
La difusión también es diferente, como se ha visto anteriormente. Hay que dar a conocer estos documentos al ciudadano y a los investigadores mediante muy diversos métodos: la reproducción del documento para investigación, preservación y difusión, la publicación de trabajos de investigación sobre los fondos del centro y, especialmente, las exposiciones, tanto en el mismo centro como el préstamo para exposiciones externas, son actuaciones que se han mostrado muy eficientes. Todas estas actividades exigen conocimientos relativos a seguros, sobre aspectos técnicos de la edición, de la reproducción digital... (Mouren, 2012).

De la misma manera que hay que prever que el conjunto de actuaciones llevadas a cabo en la biblioteca para disponer un libro en un estante a la espera de su uso tiene que adecuarse al máximo grado de atención a su conservación, la metodología del uso del documento por parte del investigador (incluida la suspensión del uso de un ejemplar por parte de los investigadores), los métodos de difusión (como la denegación de la exposición de un ejemplar), etc., también se han de sujetar a la preservación del documento como patrimonio y como constituyente de un conjunto constitutivo del centro. Por esta causa, las decisiones adoptadas por los especialistas en preservación se convierten en vinculantes y preferentes sobre las que puedan tomar los propios bibliotecarios en numerosas actividades: tiempos de exposición, uso en sala, almacenamiento... (Tacón-Clavaín, 2010a; 2010b; 2011a; 2011b).

Los bienes patrimoniales sólo se pueden comprender en su integridad y adquirir pleno sentido si están contextualizados

\section{La biblioteca}

También se ha de tener en cuenta un principio básico: si un objeto patrimonial se analiza en su contexto, aumenta el conjunto de valores que atesora, porque los bienes patrimoniales sólo se pueden comprender en su integridad y adquirir su pleno sentido si están contextualizados.

El documento, salvo que no se haya utilizado ni movido del lugar en el que se depositó tras su fabricación (lo que lo individualizaría por extraño), posee una "vida" y, en su paso de propietario en propietario, de lector en lector, y de depósito documental en depósito documental, se van produciendo interacciones entre el lector, el propietario, el depósito documental y el documento, llegando al momento actual, tras estas actuaciones, modificado y frecuentemente relacionado con un contexto específico. El análisis de esta trayectoria vital del libro es esencial para reconocer la historia del ejemplar y la de las bibliotecas. Si se extrae el ejemplar de ese contexto, se pierde la posibilidad de identificar los factores que intervinieron en hacer que el documento sea en la actualidad como es. Además, cada uno de estos ejemplares ha tenido una función y un porqué dentro de una colección. De esta manera, se puede hablar de una relación íntima entre el documento y la colección que determina la mutación de ambos mediante el acomodo del uno en la otra y de la colección como receptora del documento. 
La legislación establece una división de gran interés con respecto al patrimonio bibliográfico: las unidades documentales que poseen unas características especiales se incluyen de forma directa dentro del conjunto de bienes patrimoniales, pero también se incluyen los conjuntos bibliográficos pertenecientes al ámbito público tengan o no esas características. El responsable del patrimonio debe entender que el contexto, el conjunto, la colección (o mejor la biblioteca) es un único bien patrimonial indivisible que se comprende y se entiende así, reunida, preferiblemente en el ámbito espacial en el que se desarrolló. Ya no se trata de analizar el conocimiento almacenado en una obra específica, por muy puntera que sea, ni de analizar una signo de propiedad, ni de observar una bella encuadernación o un grabado determinado; se trata de ver, conocer y comprender qué características posee una biblioteca conventual en el siglo XVIII en un espacio determinado, qué lee la nobleza en el XVIII o qué interesó y conformó la biblioteca de un médico en el XVI, qué otros objetos poseía unidos a la biblioteca, qué autores leía, en qué idiomas...

Para tratar correctamente el libro antiguo, el bibliotecario y el investigador deben contar con una bibliografía de apoyo que les ayuden en su labor

Existen bibliotecas que adoptan de forma consciente una posición museográfica conjugándola con la propia bibliotecaria. Son perfectamente conocidas e identificables esas bibliotecas que se pueden denominar bibliotecas-museo. En ellas el contexto y el continente se imbrican en un todo que se trasforma en el objeto de estudio y de difusión. La protección patrimonial se amplía a todos los elementos constitutivos de la biblioteca. En estos casos la extracción de alguno de los componentes representa una verdadera amputación de valores patrimoniales, por mucho que se pueda percibir el interés del conjunto. Es evidente que el mostrar "en su contexto" los materiales patrimoniales resulta notablemente complicado en el caso de los libros antiguos, ya que prima la conservación, pero en los casos en los que ha sido posible mantener un nivel de conservación aceptable, teniendo presente que los fondos existentes en la actualidad son también fruto de una historia, es lo deseable y donde se ha de poner el máximo esfuerzo.

Trasciende, por tanto, la información puntual contenida en uno o en varios volúmenes; su alcance (que bien puede identificarse con el conjunto de valores) se ha expandido al conjunto de documentos en su medio ambiente, siendo éste el que hay que proteger. He aquí una nueva relación entre las bibliotecas patrimoniales y los museos: en ellos los objetos patrimoniales se analizan y muestran, siempre que se puede, en su contexto.

No se deberían confundir las bibliotecas patrimoniales, ni siquiera las bibliotecas-museo con los denominados museos del libro o de la imprenta: no sólo las finalidades, sino también los objetos y conocimientos que se pretende trasmitir son completamente diferentes. Los museos de la imprenta y del libro muestran cómo se hace el libro, como dice Grego- rio: "un museo del libro nasce per esporre al pubblico questo singolare 'oggetto', mostrandone le più segrete sfaccettature e il progressivo divenire nel tempo; portando sotto i riflettori le tecniche di produzione e le innovazioni grafiche, i protagonisti della storia editoriale e le trasformazioni avvenute nelle pratiche di scrittura, di lettura e di mercato" (2006). Baste como ejemplo el de la Comunidad Valenciana situado en El Puig, mientras que las bibliotecas históricas o patrimoniales muestran cómo era el conocimiento en un momento dado.

Un objeto de estudio que presenta esta cierta complejidad exige que el bibliotecario y el investigador tengan acceso a una bibliografía de apoyo, y a unas herramientas de trabajo que les ayuden en su labor, de acceso directo y sin préstamo, en lo que Carrión-Gútiez (1998, p. 37) denominó como "biblioteca de presencia" (Prasenzbibliothek). La identificación de una edición, de un estilo en la encuadernación, un ex-libris, una firma, la confirmación de la integridad del ejemplar, la existencia de una falsificación, la facsimilización de una página o páginas, la identificación de un pastiche... son aspectos que se deben conocer antes de proceder al tratamiento del volumen concreto. La adquisición de un ejemplar requiere conocer la existencia de otros en la proximidad, las características del mismo, la licitud de su procedencia, la adecuación de su precio al precio de mercado... El préstamo para una exposición sólo se puede realizar si previamente se han evaluado los riesgos (de transporte, de exposición, de reproducción para el catálogo...), se ha estudiado el precio del ejemplar, se ha analizado su valor para la biblioteca y la posibilidad de una reposición en caso de pérdida... Estas actividades requieren unas herramientas, unos instrumentos imprescindibles que conforman una biblioteca de apoyo, que suele ser de uso compartido por parte de los bibliotecarios y de los investigadores, que también precisan estas mismas herramientas. Este fondo contiene también aquellas obras que tratan sobre el centro y sus colecciones, sobre su especialización... por una parte; y sobre el libro antiguo en general, su confección, evolución y estructura... por otra. Fondos similares para el apoyo a los investigadores y técnicos son imprescindibles también en los museos.

\section{El contexto es fundamental para com- prender la unidad bibliográfica}

En esa conjugación de las bibliotecas con fondos patrimoniales y los museos, se hallan otras identidades: cada vez más las grandes bibliotecas cuentan con tiendas especializadas como los museos y, también, cada vez más, se pueden encontrar museos especializados en las bibliotecas que presentan exposiciones permanentes y temporales que tienen como objeto principal los fondos y colecciones de las propias bibliotecas.

\section{A modo de conclusión}

Las similitudes entre las bibliotecas con fondos antiguos y los museos son notables, existen muchos lugares comunes y muchos puntos de proximidad. La separación en el mundo académico no debe ser obstáculo para propiciar la bús- 
queda de sinergias de las que ambos mundos pueden salir beneficiados.

El objeto de trabajo de ambas instituciones pertenece al ámbito patrimonial, lo que ofrece como resultado fines $y$ objetivos similares. De hecho, el documento antiguo como documento de investigación o consulta presenta características específicas propias diferentes de las de los libros modernos.

El libro antiguo debido a las vicisitudes de su elaboración y el transcurso del tiempo deviene en ejemplar único, perfectamente diferenciable de los que con él formaron la edición $y$, por ello, identificado dentro de la colección a la que pertenece. Esta situación se produce igualmente en los objetos conservados en el museo.

De la misma manera que ocurre cuando los objetos custodiados en museos poseen referentes modernos, las diferencias que existen entre los documentos impresos antiguos y los contemporáneos exigen un profesional que posea una especialización en este tipo de fondos.

El libro antiguo requiere un proceso de identificación imprescindible ya que es preciso conocer qué es exactamente lo que se tiene delante para poder llevar a cabo un tratamiento correcto. Este mismo proceso identificativo se produce con las piezas museísticas. Son procesos que afectan a diversos aspectos del libro: textual, material, procedencia...

El contexto es fundamental para comprender la unidad bibliográfica, de hecho, en algunas ocasiones lo que se posee no es un libro sino una biblioteca completa, en cuyo caso se amplía la protección a todo el conjunto y al continente. El contexto es esencial como en el resto de los objetos patrimoniales.

Como se puede observar, en casi todas las particularidades mencionadas se encuentran similitudes con los museos: preponderancia de la preservación, identificación de los documentos, similitud de los mercados anticuarios, necesidad de la identificación previa, convergencia de las técnicas y métodos de difusión de los fondos.

\section{Nota}

1. Magritte pintó una pipa y debajo escribió “Ceci n'est pas une pipe" ("esto no es una pipa"), lo que parece una contradicción, pero es realmente cierto: la pintura no es una pipa, es la imagen de una pipa. Cuando a Magritte le preguntaron sobre esta imagen, respondió: "por supuesto no es una pipa: sólo trate usted de llenarla con tabaco".

\section{Bibliografía}

Arjona, Martha (2003). Patrimonio cultural e identidad nacional. 2a ed. La Habana: Boloña. ISBN: 9597126133

Abranches, Henrique (1988). Identidad y patrimonio cultural. La Habana: Ciencias Sociales.

Bos, Jan (2012). "All books are equal, but some books...: towards a modern vision of special collections". En: Mouren, Raphaële (ed.). Ambassadors of the book: competences and training for heritage librarians. The Hague: International Federation of Library Association and Institutions, De
Gruyter, Saur. (IFLA Publications, 160), pp. 15-23. ISBN: 978 3110301274

Carrión-Gútiez, Manuel (1998). "Tres lecciones sobre la Biblioteca Nacional de España”. En: Rodríguez-Álvarez, Ramón; LlordénMiñambres, Moisés (eds.). El libro antiguo en las bibliotecas españolas. Oviedo: Universidad de Oviedo, Servicio de Publicaciones, pp. 9-38.

Cataluña (1993). "Ley 9/1993, de 30 de septiembre, del Patrimonio cultural catalán". Diari oficial de la Generalitat de Catalunya, n. 1807, pp. 6.748-6.758.

http://portaldogc.gencat.cat/utilsEADOP/PDF/1807/46350. $p d f$

http://noticias.juridicas.com/base_datos/CCAA/ca-19-1993. html

Clemente, Yolanda (1993). "Análisis, identificación y descripción analítica del libro antiguo: las ediciones y sus variantes: emisiones y estados". En: Pedraza-Gracia, ManuelJosé, Clemente-San-Román, Yolanda; De-los-Reyes-Gómez, Fermín. El libro antiguo. Madrid: Síntesis, pp. 249-261. ISBN: 8497561538

Gregorio, María (2006). Imago libri. Musei del libro in Europa. Milano: Sylvestre Bonnard. ISBN: 8889609176

La Rioja (2004). "Ley 7/2004, de 18 de octubre, de Patrimonio cultural, histórico y artístico de La Rioja". Boletín oficial de La Rioja, n. 136.

http://goo.gl/X4qoi

Martín-Abad, Julián (2004a). Los libros impresos antiguos. Valladolid: Universidad de Valladolid. ISBN: 9788484482796

Martín-Abad, Julián (2004b). La valoración del libro: el punto de vista del bibliotecario de fondo antiguo. Documentos de trabajo UCM. Biblioteca Histórica, n. 8. pp. 1-25.

http://eprints.ucm.es/5698

Moll, Jaime (1979). "Problemas bibliográficos del libro del Siglo de Oro". Boletín de la Real Academia Española, v. 59, n, 216, pp. 49-107.

http://www.cervantesvirtual.com/obra/problemasbibliograficos-del-libro-del-siglo-de-oro--0

Mouren, Raphaële (ed.) (2012). Ambassadors of the book: competences and training for heritage librarians. The Hague: International Federation of Library Association and Institutions, De Gruyter, Saur (IFLA Publications, 160). ISBN: 978110301274

Navarra (2005). "Ley foral 14/2005, de 22 de noviembre, del patrimonio cultural de Navarra". Boletín oficial de Navarra, n. 141. http://www.lexnavarra.navarra.es/detalle.asp? $r=4509$

País Vasco (1990). "Ley 7/1990, de 3 de julio, de patrimonio cultural vasco". Boletín oficial del País Vasco. 157, pp. 70627092.

http://goo.gl/sR7HW

Pearson, David (1998). Provenance research in book history: a handbook. London: The British Library. ISBN: 0712303448

Pearson, David (2012). Books as history: the importance of books beyond their texts. New Castle, Oak Knoll Press. ISBN: 9781584563150 
Pedraza-Gracia, Manuel-José (2008). “La gestión del patrimonio bibliográfico español y el catálogo colectivo de patrimonio bibliográfico: un ejemplo de interrelación entre la administración y el ciudadano". En: Rover, Aires José (ed). Inclusão digital e governo eletrônico. Zaragoza: Prensas Universitarias de Zaragoza, Lefis series v. 3, n. 9, pp. 217-246. http://zaguan.unizar.es/record/4170

Perú (2004). Ley general del patrimonio cultural de la nación. Ley n. 28296.

http://www.ilam.org/ILAMDOC/ley28296\%20\%20380.pdf

Prats, Llorenç (1997). Antropología y patrimonio. Barcelona, Ariel. ISBN: 97884344422117

Racedo, Josefina; Requejo, María-Isabel; Segura, Zulma; Taboada, María-Stella (2004). Patrimonio cultural e identidad. Buenos Aires: Cinco/Cercapu. ISBN: 9509693685

Richard, Hélène (2010). La formation aux questions patrimoniales dans les bibliothèques. Paris: Ministère de l'Enseignement Supérieur et de la Recherche. Rapport n. 2010-016.

http://media.enseignementsup-recherche.gouv.fr/ file/2010/52/1/Formation_aux_questions_patrim_ def_166521.pdf

Santos-Aramburo, Ana; Torres-Santo-Domingo, Marta (2004). "La Biblioteca Histórica de la Universidad Complutense: una primera aproximación a sus procedencias": En: La memoria de los libros: estudios sobre la historia del escrito y de la lectura en Europa y América. Salamanca: Instituto de Historia del Libro y la Lectura, Tomo II, págs. 265-286. ISBN: 9788493350444

http://eprints.ucm.es/9836/1/Procedencias_BH.pdf
Serrai, Alfredo (1992), "Le biblioteche storiche". I/ bibliotecario, n. 33/34, pp. 1-11.

Tacón-Clavaín, Javier (2010a). “Cajas de conservación para libros". Documentos de trabajo UCM. Biblioteca Histórica, 2010/4. http://eprints.ucm.es/10637

Tacón-Clavaín, Javier (2010b). "El uso de atriles para la apertura de libros antiguos". Documentos de trabajo UCM. Biblioteca Histórica, 2010/5.

http://eprints.ucm.es/10638

Tacón-Clavaín, Javier (2011a). "Procedimiento para la manipulación de códices manuscritos durante la consulta". Documentos de trabajo UCM. Biblioteca Histórica, 2011/6.

http://eprints.ucm.es/12460

Tacón-Clavaín, Javier (2011b). "Procedimiento para la apertura de libros intonsos de fondo antiguo". Documentos de trabajo UCM. Biblioteca Histórica, 2011/7.

http://eprints.ucm.es/12472

Tugores, Francesca; Planas, Rosa (2006). Introducción al patrimonio cultural. Gijón: Trea. ISBN: 8497042425

Unesco (2000). Definitions for "intangible cultural heritage". Member states (Replies to questionnaires sent to national commissions in February and August 2000). Paris: Unesco. http://www.unesco.org/culture/ich/doc/src/00078-EN.pdf

Unesco (2009). Lista representativa del patrimonio cultural inmaterial de la humanidad. París: Unesco.

Venezuela (1993). "Ley de protección y defensa del patrimonio cultural". Gaceta oficial de la República de Venezuela, n. 4.623 extraordinario.

\section{Próximos temas centrales}

Noviembre 2013

Enero 2014

Marzo 2014

Mayo 2014

Julio 2014

Septiembre 2014

Noviembre 2014

\section{Formación y aprendizaje}

Documentación audiovisual

Políticas de información

Visualización de información

Humanidades digitales

Big data y analítica web

Marketing

Los interesados por favor consulten detenidamente las Normas para autores:

http://www.elprofesionaldelainformacion.com/autores.html

y luego envíen sus artículos a través del gestor de manuscritos OJS de la plataforma del Recyt:

http://recyt.fecyt.es/index.php/EPI/index 